\title{
Influence of co- and counter-propagating light on the phase-mismatch effect in semiconductor optical amplifiers
}

\begin{abstract}
The phase-mismatch effect, caused by the difference in confinement factor between transverse electric (TE) and transverse magnetic (TM) modes, has not been observed in semiconductor optical amplifiers (SOAs) and is not typically accounted for. In this work, we investigate the characteristics of the phase-mismatch four wave mixing (FWM) effect, which occurs in SOA devices. Our results reveal a sinc-like response in the intensity of co- and counter-FWM conjugates as a function of the detuning shift between interacting beams. It is demonstrated that the coherence lengths between the phase match/mismatch cycles differ between co- and counter-propagating nondegenerate FWM experiments and depend on the amplified TE/TM polarizations of interacting waves aligned with respect to the birefringent axes of SOAs. The coherence lengths between each phase match/mismatch cycle in co-propagation experiments are found to be 1600 and infinite $\mathrm{GHz}$, respectively, compared to 800 and $400 \mathrm{GHz}$ found in counter-propagation experiments.
\end{abstract}

Keyword: Coherence length; Four-wave mixing; Phase match/mismatch; Semiconductor optical amplifiers 